\title{
A nonlinear controller for pneumatic servo systems: Design and experimental tests
}

\author{
S. Riachy and M. Ghanes
}

\begin{abstract}
This paper is dedicated to the problem of pneumatic cylinder control without pressure measurement. Based on the theory of homogeneous, finite time stable, ordinary differential equations, a state feedback nonlinear controller is proposed. The closed loop system stability is proved and an attraction domain of the controller is given. The performances and the effectiveness of the proposed controller are illustrated against an experimental setup consisting of a pneumatic cylinder controlled by dSPACE dS1103 microcontroller.
\end{abstract}

\section{INTRODUCTION}

Pneumatic actuators are often used in industrial systems due to their high power-to-weight ratio, ease of maintenance and safety. However, they present highly nonlinear dynamics mainly due to the compressibility of air, friction forces and asymmetry of the air pressure forces in the cylinder compartments. Although the piston dynamics is simple since it corresponds to a double integrator, the mathematical model of the whole actuator is highly uncertain since the air pressure dynamics is often based on empirical considerations [17]. The complexity of the model increases as one wants to model friction forces [6] since they introduce nonlinearities of discontinuous type. Consequently, a controller for pneumatic systems has to deal with uncertainties and hard nonlinearities.

The control of pneumatic actuators has received an abundant literature. PID controllers, fuzzy controllers, model/nonmodel based controllers, state/output based controllers, linear/nonlinear controllers has been investigated [5], [10], [12], [13], [14], [15], [16], [18], [20]. In what follows, some works that are directly related to the present one are summarized.

\section{A. Related works}

In [20], a practical control strategy was developed based on the piston position measurement. The authors investigated the pressure dynamics and revealed a close relation between pressure feedback and acceleration feedback. This investigation motivated the design of a PID controller augmented with an acceleration feedback. The controller features conceptual simplicity and low computational cost. Velocity and acceleration were estimated by differentiating the output signal.

In [18], a sliding mode control was designed. It is based on the concept of multiple surfaces and admit a backstepping design structure while featuring robustness properties of sliding modes. In addition to its robustness to uncertainties,

S. Riachy and M. Ghanes are with ECS-Lab/ENSEA, France. e-mail: riachy@ensea.fr, ghanes@ensea.fr, Tél: 0033130736638

S. Riachy is also with Non-A team, INRIA Lille-Nord-Europe. the controller permit to cope with mismatched perturbations ${ }^{1}$ caused by unknown forces acting on the piston rod. The stability of the closed loop has been proven, however, the structure of the controller is quite complex.

In [5], two conventional, first order, sliding mode controllers were developed, the first one is based on the linearized model while the second one is based on the nonlinear model. Good performances were obtained in stabilization and in tracking. The experiments were conducted with the cylinder mounted in a vertical and a horizontal positions with different loads. The equivalent control method (introduced by V.I. Utkin [19]) was implemented by using pressure sensors. It should be noticed that the control in the vertical position is more challenging due to the asymmetry induced by the weight of the payload.

In [14], a backstepping construction was developed based on Lyapunov functions. The controller uses pressure sensors. It performs well on a horizontally mounted cylinder.

In [13], a pressure observer was designed based on a linearized model of the system. A state feedback controller as well as an output (observer based) feedback controller were compared in order to evaluate the effectiveness of the observer.

In [12], a sliding mode control was developed to control a pneumatic cylinder using solenoid valves. The control is interesting since it provides accurate positioning of a horizontally mounted cylinder while using a low cost technology of valves.

\section{B. Main contributions}

The present work proposes a simple controller which is based on the concepts of homogeneity and finite time stability [2], [3], [4]. To the best of the authors knowledge, these concepts has not yet been investigated in the context of pneumatic actuators. The proposed controller involves a second order constraint and has some resemblance with sliding mode based controllers [5], [12]. Recall that a major drawback of sliding mode control is the chattering effect caused by the discontinuous input. The proposed controller involves a tuning parameter $\alpha \in[0,1]$ such that setting $\alpha=1$ turns out the controller to a linear one while setting $\alpha=0$ results in a conventional sliding mode controller. Naturally, setting $\alpha$ close to zero provides a continuous approximation of the discontinuous control. From a theoretical point of view, if $\alpha>0$, the proposed controller do not suffer from chattering however, in a practical context a chattering may appear caused by a noisy measurement. In summary, the tuning of $\alpha$ permits to ally the robustness against perturbations of sliding modes to the smoothness of the linear control.

\footnotetext{
${ }^{1}$ Perturbations that do not enter via the range space of the control effort.
} 
The stability of the closed loop is proved and a domain of attraction of the controller is evaluated. We mention that in all the previously cited works, the domain of attraction of the developed controllers was not provided. Notice that due to physical constraints on the piston displacement and on admissible velocities and accelerations imposed by the constructor, it is important to guarantee that during the transients, the system states do not leave a predefined domain. A numerical evaluation of the domain of attraction for the pneumatic actuator that served in the experimental validation is given. It shows that the calculated domain is plausible. In addition, the attraction domain is experimentally validated.

In the experimentation section VI, the control signals are provided in order to illustrate the influence of the parameter $\alpha$ and show that a proper tuning combines robustness and chattering reduction. We mention that, except for [12], the control signals were not reported in previous works.

As in [20], the controller uses the piston position and its first and second derivatives and do not rely on pressure measurements. The estimation of the output derivatives is accomplished by using the numerical differentiators introduced in [11]. They consist of finite impulse response filters and feature ease of implementation and low computational cost. Velocity and acceleration estimates are provided and shown to evolve in the predefined domain.

The experimental validation is accomplished on a vertically mounted cylinder with a payload ranging from $0 \%$ to $75 \%$ of the maximal admissible load.

\section{Paper organization}

The paper is organized as follows. Section II briefly introduces homogeneous, finite-time-stable systems. The dynamic model is recalled in section III while the controller is developed in section IV. The position signal derivatives are estimated in section $\mathrm{V}$. The experiments are presented in section VI. The paper ends in section VII with a conclusion.

\section{HomogeneOUS SYSTEMS AND FINITE TIME CONVERGENCE}

\section{A. A brief summary}

The present section summarizes elementary notions about homogeneous, finite time stable, ordinary differential equations. Only the case of a scalar system is recalled since it is used in forthcoming sections. Section II-A is mainly taken from [3]. Interested readers can see [2], [4] for deeper studies on the subject. In the sequel, the following notation is used:

$$
\Phi_{\alpha}(x)=\operatorname{sign}(x)|x|^{\alpha}, x \in \mathbb{R}, \alpha>0
$$

where $|\bullet|$ denotes the absolute value of $x$. Using (1), the following rules for derivatives are verified, except at $x=0$, where the derivatives are not defined: $\frac{d \Phi_{\alpha}(x)}{d x}=\alpha|x|^{\alpha-1}$ and $\frac{d|x|^{\alpha}}{d x}=$ $\alpha \Phi_{\alpha-1}(x), \quad \forall x \in \mathbb{R} \backslash\{0\}$. Note also that $\Phi_{\alpha}(x) \times \Phi_{\beta}(x)=$ $|x|^{\alpha+\beta}$. The mapping defined by $x \mapsto \Lambda_{r} x$, where $\Lambda_{r} x=\lambda^{r} x$, is called a dilation with $r$ and $\lambda$ two positive numbers. A function $f(x): \mathbb{R} \mapsto \mathbb{R}$ is homogeneous with degree $m \in \mathbb{R}$ with respect to the dilation $\Lambda_{r}$ if $f\left(\Lambda_{r} x\right)=\lambda^{r+m} f(x) ; \forall \lambda>$ $0, \forall r>0, x \in \mathbb{R}$. Consequently, the scalar ordinary differential equation $\dot{x}=f(x)$ is said to be homogeneous with degree $m$ with respect to $\Lambda_{r}$. A remarkable property of homogeneous systems with negative degree (i.e $m<0$ ) (see [4]) is the finite time stability of an equilibrium point if this equilibrium is asymptotically stable.

The simple integrator

$$
\dot{x}=-\Phi_{\alpha}(x), \alpha \in(0,1),
$$

is of main importance for the control design in the sequel. It is homogeneous with negative degree $m=r(\alpha-1)$ with respect to $\Lambda_{r}$ and is therefore finite time stable. In addition, if one takes $\alpha=0,(2)$ reduces to $\dot{x}=-\operatorname{sign}(x)$ resulting in a conventional variable structure system featuring also finite time stability [19]. Moreover, for $\alpha=1$ one obtains $\dot{x}=-x$, a linear, asymptotically stable, system. Therefore, system (2) with $\alpha$ small enough can be seen as a continuous approximation of a variable structure system. It features the finite time stability property, shares good robustness with sliding modes and provides a continuous, chattering free, system in theory.

From a theoretical point of view, finite time stability provides a decisive advantage compared to asymptotic stability. However, in our practical context, the homogeneous controller is designed on a subsystem of the pneumatic actuator equations after a nonlinear transformation. A zero dynamics remains which is asymptotically stable. In sum, the developed state feedback controller provides asymptotic stabilization of the pneumatic system with capability to ally robustness and chattering reduction through a parameter tuning $(\alpha)$.

\section{B. Application to the stabilization of nonlinear systems}

Consider a nonlinear system:

$$
\dot{\xi}=f(\xi)+g(\xi) u
$$

where $\xi \in \mathbb{R}^{n}, f(\xi): \mathbb{R}^{n} \rightarrow \mathbb{R}^{n}$ and $g(\xi): \mathbb{R}^{n} \rightarrow \mathbb{R}^{n}$ are nonlinear functions and $u \in \mathbb{R}$.

Assume that $S$ is a given constraint, the zeroing of which solves the stabilization problem of (3), that is, if $S=0$, $\xi\left(t, t_{0}, \xi_{0}\right) \rightarrow 0$ as $t \rightarrow \infty$. Assume that the relative degree of $S$ is one and that its time derivative can be written in the following form: $\dot{S}=f_{1}(S)+f_{2}(S) u+p(t, S)$. $f_{1}(S)$ is a Lipschitz continuous function $\left|f_{1}(S)\right|<L S$ where $L$ is a positive constant. $f_{2}(S)$ is a nonlinear function bounded away from zero that is there exists $f_{2}$, a strictly positive constant, such that $f_{2}(S)>\underline{f}_{2} \cdot p(t, S)$ is a bounded perturbation. Then, if $p(t, S)$ is sufficiently small, there exist a ball $B_{\delta}$ around the origin $(S=0)$ with radius $\delta$, a positive constant, such that the control $u=-\Phi_{\alpha}(S), 0<\alpha<1$, ensures the convergence of $S$ inside $B_{\delta}$. Consequently, $x$ converges inside a "small" ball around the origin $x=0$. In addition, if $p(t, S)=0$, the system is asymptotically stable at zero. This procedure is developed for the pneumatic actuator in the coming sections.

\section{DYNAMIC MODEL OF THE SYSTEM}

The derivation of the dynamic equations of the pneumatic actuator can be found in [17], [18] and [20]. The state vector is of dimension 4 . With $y$ to denote the position of the piston, 


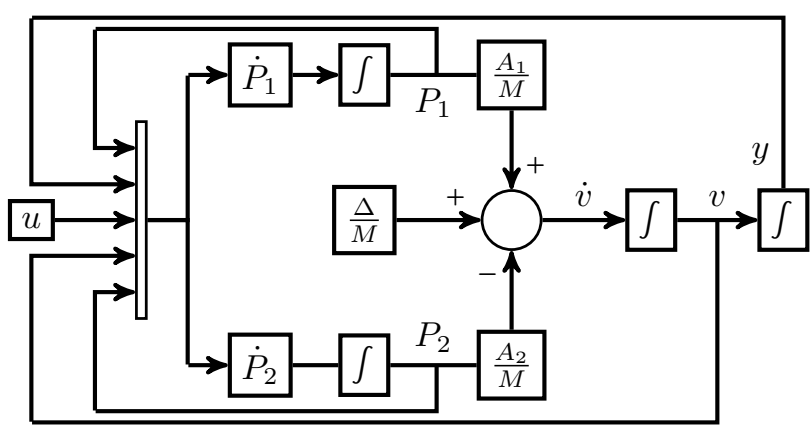

Fig. 1. Bloc diagram of the system model

$v$ its velocity, $P_{1}$ and $P_{2}$ the pressures in both compartments of the cylinder, the dynamic equations writes:

$$
\begin{aligned}
\dot{y} & =v \\
\dot{v} & =M^{-1}\left(P_{1} A_{1}-P_{2} A_{2}+\Delta\right) \\
\dot{P}_{1} & =-\frac{k P_{1} v}{L+y}+\frac{\Omega}{A_{1}}\left\{\frac{1+\sigma(u)}{2} \frac{\gamma_{1 b} P_{s}}{L+y}-\frac{1-\sigma(u)}{2} \frac{\gamma_{1 e} P_{1}}{L+y}\right\}|u| \text { (4) } \\
\dot{P}_{2} & =\frac{k P_{2} v}{L-y}+\frac{\Omega}{A_{2}}\left\{\frac{1-\sigma(u)}{2} \frac{\gamma_{2 b} P_{s}}{L-y}-\frac{1+\sigma(u)}{2} \frac{\gamma_{2 e} P_{2}}{L-y}\right\}|u|, \quad \text { (5) }
\end{aligned}
$$

where $\Omega=k \sqrt{R k T} A_{o} / U, \sigma(u)$ denotes the signum function ${ }^{2}$, $A_{o}$ the area of the orifice of the servovalve. The control $u$ represents the input voltage to the servovalve while $U$ denotes the maximal input voltage $(|u| \leq U) . P_{s}$ denotes the supply pressure. $\Delta$ represents an external load and can also represent gravity when the cylinder is mounted in a vertical position. $T$ denotes the air temperature, assumed to be constant. The heat coefficient for air is $k$, while $R$ is the perfect gas constant. $2 L$ denotes the total length of the cylinder while $M$ the mass of the moving part (piston, payload etc.). $\gamma_{1 b}, \gamma_{2 e}, \gamma_{1 e}$ and $\gamma_{2 b}$ are given by: $\gamma_{1 b}=\sqrt{\frac{2}{k-1}}\left(\frac{P_{1}}{P_{s}}\right)^{\frac{k+1}{2 k}} \sqrt{\left(\frac{P_{1}}{P_{s}}\right)^{\frac{1-k}{k}}-1}$ if $\frac{P_{1}}{P_{s}} \geq 0.528$, $\gamma_{1 b}=0.58$ if $\frac{P_{1}}{P_{s}}<0.528, \gamma_{2 e}=\sqrt{\frac{2}{k-1}}\left(\frac{P_{a}}{P_{2}}\right)^{\frac{k+1}{2 k}} \sqrt{\left(\frac{P_{a}}{P_{2}}\right)^{\frac{1-k}{k}}-1}$ if $\frac{P_{a}}{P_{2}} \geq 0.528, \gamma_{2 e}=0.58$ if $\frac{P_{a}}{P_{2}}<0.528, \gamma_{1 e}=$ $\sqrt{\frac{2}{k-1}}\left(\frac{P_{a}}{P_{1}}\right)^{\frac{k+1}{2 k}} \sqrt{\left(\frac{P_{a}}{P_{1}}\right)^{\frac{1-k}{k}}-1}$ if $\frac{P_{a}}{P_{1}} \geq 0.528, \gamma_{1 e}=0.58$ if $\frac{P_{a}}{P_{1}}<0.528, \gamma_{2 b}=\sqrt{\frac{2}{k-1}}\left(\frac{P_{2}}{P_{s}}\right)^{\frac{k+1}{2 k}} \sqrt{\left(\frac{P_{2}}{P_{s}}\right)^{\frac{1-k}{k}}-1}$ if $\frac{P_{2}}{P_{s}} \geq 0.528$, $\gamma_{2 b}=0.58$ if $\frac{P_{2}}{P_{s}}<0.528 . P_{a}$ denotes atmospheric pressure. A bloc diagram of the open loop system is depicted in Figure 1. With $F=P_{1} A_{1}-P_{2} A_{2}$, the resultant pressure force, the system model can be reduced to (see [18]):

$$
\begin{aligned}
\dot{y} & =v \\
\dot{v} & =M^{-1}(F+\Delta) \\
\dot{F} & =-\left(\frac{P_{1} A_{1}}{L+y}+\frac{P_{2} A_{2}}{L-y}\right) k v+G\left(y, P_{1}, P_{2}\right) u
\end{aligned}
$$

where $\quad G\left(y, P_{1}, P_{2}\right)=\Omega\left[\frac{1+\sigma(u)}{2}\left(\frac{\gamma_{1 b} P_{s}}{L+y}+\frac{\gamma_{2 e} P_{2}}{L-y}\right)\right.$ $\left.+\frac{1-\sigma(u)}{2}\left(\frac{\gamma_{1 e} P_{1}}{L+y}+\frac{\gamma_{2 b} P_{s}}{L-y}\right)\right]$.

Consider the invertible change of coordinates $y=y, v=$ $v$ and $S=M^{-1}(F+\Delta)+a v+b y$. Its inverse is given by $F=M(S-a v-b y)-\Delta$. The jacobian matrix $\mathcal{J}$, associated

${ }^{2} \sigma(u)=+1$ if $u>0, \sigma(u)=0$ if $u=0$ and $\sigma(u)=-1$ if $u<0$ to this change of coordinates $\mathcal{J}=\left[\begin{array}{ccc}1 & 0 & 0 \\ 0 & 1 & 0 \\ -b M & -a M & M\end{array}\right]$ is of full rank (since $M>0$ ) and consequently is invertible. Therefore, the change of coordinate is a diffeomorphism. In $(y, v, S)$ coordinates the system equations are given by:

$$
\begin{aligned}
\dot{y} & =v \\
\dot{v} & =-a v-b y+S \\
\dot{S} & =M^{-1}\left[-\left(\frac{P_{1} A_{1}}{L+y}+\frac{P_{2} A_{2}}{L-y}\right) k v+G\left(y, P_{1}, P_{2}\right) u\right] \\
& +M^{-1} \dot{\Delta}+a(S-a v-b y)+b v
\end{aligned}
$$

Since a main contribution of the paper is to provide a domain of attraction for the designed controller, and in order to show that the bounds are physically plausible, the physical parameters of the pneumatic cylinder that served in the concrete validation are listed in table I.

\begin{tabular}{|c|c|}
\hline Cylinder total length: $2 \mathrm{~L}$ & $0.2 \mathrm{~m}$ \\
\hline Piston area: $A_{1}$ & $0.0008042 \mathrm{~m}^{2}$ \\
\hline Piston area: $A_{2}$ & $0.0007257 \mathrm{~m}^{2}$ \\
\hline Servovalve orifice area: $A_{o}$ & $1.26 \times 10^{-5} \mathrm{~m}^{2}$ \\
\hline Heat coefficient for air: $k$ & 1.4 \\
\hline Perfect gas constant: $R$ & $286.987 \mathrm{~J} / \mathrm{kgK}$ \\
\hline Air temperature: $T$ & $293 \mathrm{~K}$ \\
\hline Supply pressure: $P_{s}$ & $4 \times 10^{5}$ Pascals \\
\hline Atmospheric pressure: $P_{a}$ & $1 \times 10^{5}$ Pascals \\
\hline Maximum input voltage: $U$ & $1 \mathrm{volt}$ \\
\hline Maximal admissible load in vertical position & $15 \mathrm{Kg}$ \\
\hline Compressor input power & $0.13 \mathrm{KW}$ \\
\hline
\end{tabular}

TABLE I

SYSTEM PARAMETERS

\section{Control Design}

Before proceeding to the control design, it is important to notice that the control gain $G\left(y, P_{1}, P_{2}\right)$ can be zero whenever $\left(P_{1}, P_{2}\right)=\left(P_{s}, P_{a}\right)$ or $\left(P_{1}, P_{2}\right)=\left(P_{a}, P_{s}\right)$. Any of these two situations result in a control singularity. Fortunately, they barely happen in practice since they correspond to one of the following situations:

- An excessive load $\Delta$, that is $P_{s} A_{1}-P_{a} A_{2} \approx \Delta$ or $-P_{a} A_{1}+P_{s} A_{2} \approx \Delta$.

- An excessive acceleration, if $\Delta$ is small enough, which may lead to the piston hitting the stops.

Assume now that there exists a control guaranteeing that the acceleration cannot exceed predefined bound. With an acceptable load $\Delta$, this means that the pressure difference $\left|P_{1}-P_{2}\right|$ is bounded since, from (6), one deduces that $\left|P_{1} A_{1}-P_{2} A_{2}\right| \leq|M \ddot{y}|+|\Delta|$. Moreover, by examining (4) and (5), it appears that $\dot{P}_{1}$ and $\dot{P}_{2}$ have opposite sign independently of the velocity $v$ and the control $u$. Consequently, $P_{1}$ and $P_{2}$ cannot increase or decrease simultaneously. With these two observations, one can notice that if $P_{1}$ and $P_{2}$ are properly initialized (at atmospheric pressure for example) then neither $P_{1}$ nor $P_{2}$ will exceed $P_{s}$. A rigorous proof of such an assertion is quite lengthy and is out of the scope of the present work. However, this discussion can motivate the following assumptions: 
Assumption 1: There exists a positive constant $\underline{\gamma}>0$ such that $\gamma_{1 e}>\gamma, \gamma_{1 b}>\gamma, \gamma_{2 e}>\gamma$ and $\gamma_{2 b}>\gamma$.

Assumption 2: Assume ${ }^{-}$that $\Delta^{-}<2.5 P_{a} \underline{A}$, $\underline{A}=\min \left\{A_{1}, A_{2}\right\}$.

Remark 1: The rough constant 2.5 in assumption 2 is found using the maximal admissible load for the actuator in vertical position (table I). Such a bound can be easily determined for other cylinders knowing the admissible load.

The case of a constant perturbation is investigated in section IV-A while time varying perturbations are treated in section IV-B.

\section{A. Constant perturbation $(\dot{\Delta}=0)$}

The case of a constant $\Delta(\dot{\Delta}=0)$ is considered first. The stabilizing controller and its domain of convergence are described by the following theorem.

Theorem 4.1: Let assumptions 1 and 2 be satisfied and consider $Y=[y, \dot{y}]^{\prime}$ with ' to denote the vector transpose. Take $\alpha \in(0,1)$, let $a, b$ be positive constants and $\theta>1$. Denote by $\mathcal{P}$ the positive definite matrix: $\mathcal{P}=\frac{1}{2}\left[\begin{array}{ll}b & 0 \\ 0 & 1\end{array}\right]$, and by $\Gamma$ :

$\Gamma=\frac{\theta \bar{\rho} k \bar{A}}{a \underline{\rho} \Omega \underline{\gamma}}+\frac{M L}{2 \Omega \underline{\gamma} P_{a}}\left(\frac{\theta \bar{\rho}}{a \underline{\rho}}\left(a^{2}+a b+b\right)+a\right), \quad \bar{A}=\max \left\{A_{1}, A_{2}\right\}$,

where $\bar{\rho}$ and $\rho$ are the maximal and minimal eigenvalues of $\mathcal{P}$ respectively, i.e. $\bar{\rho}=\max \{1, b\}$ and $\rho=\min \{1, b\}$. Then any trajectory of (6) initialized such that: ${ }^{3}$

$$
\left\{\begin{aligned}
\left|y_{0}\right| & <L-l, \quad 0<l<L \\
Y_{0}^{\prime} \mathcal{P} Y_{0} & <\left(\frac{\theta \bar{\rho} U}{\left.\sqrt{\frac{\rho}{\rho}} \Gamma\right)^{2}},\right. \\
\left|\ddot{y}_{0}+a \dot{y}_{0}+b y_{0}\right| & \leq \frac{U}{\Gamma},
\end{aligned}\right.
$$

converges asymptotically to zero under the control:

$$
u=-K \Phi_{\alpha}(\ddot{y}+a \dot{y}+b y)
$$

where $K=U^{1-\alpha} \Gamma^{\alpha}$ and $\Phi_{\alpha}(\ddot{y}+a \dot{y}+b y)$ uses the notation (1).

Remark 2: The first condition in (9) is imposed by the physical displacement of the cylinder. It prevents the piston from hitting the stops.

Remark 3: A bloc diagram of the closed loop system is depicted in figure 2.

Proof: Notice that the proof can be equivalently carried out using (6) or (7). Recall that $S$ is given by $S=\ddot{y}+a \dot{y}+b y$ and introduce the Lyapunov function $V_{1}(Y)=Y^{\prime} \mathcal{P} Y$. Remark that $V_{1}(Y)$ satisfies:

$$
\underline{\rho}|Y|^{2}<V_{1}(Y)<\bar{\rho}|Y|^{2} .
$$

The derivative of $V_{1}$ gives:

$$
\dot{V}_{1}(Y) \leq-|\dot{y}|(a|\dot{y}|-|S|),
$$

where $|\bullet|$ denotes a standard vector norm or the absolute value for scalar entries. With the aid of (11), inequality (12) gives $\dot{V}_{1} \leq-\frac{a}{\bar{\rho}} V_{1}+\sqrt{\frac{V_{1}}{\underline{\rho}}}|S|$, which means that $V_{1}(Y)$ decreases as long as $\sqrt{V_{1}}>\frac{\bar{\rho}}{\sqrt{\underline{\rho}}} \frac{|S|}{a}$. Consequently, (see [8]) there exists

\footnotetext{
${ }^{3}$ The constant $l$ denotes the minimal admissible distance between the piston and the stops.
}

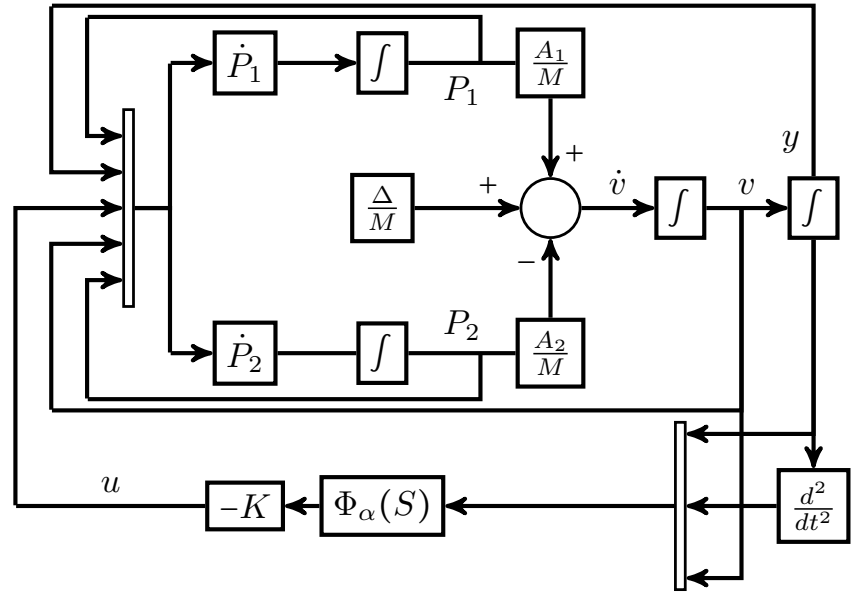

Fig. 2. Bloc diagram of the closed loop system model

$\theta>1$ and a finite amount of time such that $\sqrt{V}_{1}$ enters a $\theta$-neighborhood of $|S|$ that is:

$$
\sqrt{V_{1}} \leq \theta \frac{\bar{\rho}}{\sqrt{\underline{\rho}}} \frac{|S|}{a} .
$$

Consider now the time derivative of $S$ :

$$
\begin{aligned}
& \dot{S}=-\frac{k}{M}\left(\frac{P_{1} A_{1}}{L+y}+\frac{P_{2} A_{2}}{L-y}\right) \dot{y}+\frac{\Omega}{M}\left[\frac{1+\sigma(u)}{2}\left(\frac{\gamma_{1 b}^{\prime} P_{1}}{L+y}+\frac{\gamma_{2 e} P_{2}}{L-y}\right)\right. \\
& \left.+\frac{1-\sigma(u)}{2}\left(\frac{\gamma_{1 e} P_{1}}{L+x}+\frac{\gamma_{2 b}^{\prime} P_{2}}{L-x}\right)\right] u+a \ddot{y}+b \dot{y} . \\
& \text { where } \gamma_{1 b}^{\prime}=\sqrt{\frac{2}{k-1}\left(\frac{P_{1}}{P_{s}}\right)^{\frac{1-k}{k}}\left(\left(\frac{P_{1}}{P_{s}}\right)^{\frac{1-k}{k}}-1\right)} \text { and } \gamma_{2 b}^{\prime}= \\
& \sqrt{\frac{2}{k-1}\left(\frac{P_{2}}{P_{s}}\right)^{\frac{1-k}{k}}\left(\left(\frac{P_{2}}{P_{s}}\right)^{\frac{1-k}{k}}-1\right)} . \\
& \text { Take the Lyapunov function }
\end{aligned}
$$

$$
V_{2}(S)=\frac{1}{2} S^{2}
$$

Its time derivative reads:

$$
\begin{aligned}
\dot{V}_{2}=\frac{\Omega}{M} & {\left[\frac{1+\sigma(u)}{2}\left(\frac{\gamma_{1 b}^{\prime} P_{1}}{L+y}+\frac{\gamma_{2 e} P_{2}}{L-y}\right)+\frac{1-\sigma(u)}{2}\left(\frac{\gamma_{1 e} P_{1}}{L+y}+\frac{\gamma_{2 b}^{\prime} P_{2}}{L-y}\right)\right] S u } \\
& -\frac{k}{M}\left(\frac{P_{1} A_{1}}{L+y}+\frac{P_{2} A_{2}}{L-y}\right) \dot{y} S-a^{2} \dot{y} S-a b y S+a S^{2}+b \dot{y} S
\end{aligned}
$$

With (10), assumption 1 and $\bar{A}$ from (8), one can write the following inequality:

$$
\begin{gathered}
\dot{V}_{2} \leq-2^{\frac{\alpha+1}{2}} \frac{K}{M} \Omega \underline{\gamma}\left(\frac{P_{1}}{L+y}+\frac{P_{2}}{L-y}\right) V_{2}^{\frac{\alpha+1}{2}}+ \\
\sqrt{\frac{2}{\rho}}\left[a^{2}+a b+b+\frac{k \bar{A}}{M}\left(\frac{P_{1}}{L+y}+\frac{P_{2}}{L-y}\right)\right] \sqrt{V_{1} V_{2}}+2 a V_{2} .
\end{gathered}
$$

It is clear, according to [8] section 9.2, that there exist a finite amount of time such that the following inequality is satisfied:

$$
\sqrt{V_{1}}<\frac{\theta \bar{\rho}}{a \sqrt{\underline{\rho}}} \sqrt{2} \sqrt{V_{2}}, \theta>1 .
$$

Consequently, one obtains:

$$
\dot{V}_{2} \leq-2^{\frac{\alpha+1}{2}} \frac{K}{M} \Omega \underline{\gamma}\left(\frac{P_{1}}{L+y}+\frac{P_{2}}{L-y}\right) V_{2}^{\frac{\alpha+1}{2}}+
$$




$$
\left(\frac{2 \bar{\rho} \theta}{\underline{\rho} a}\left[a^{2}+a b+b+\frac{k \bar{A}}{M}\left(\frac{P_{1}}{L+y}+\frac{P_{2}}{L-y}\right)\right]+2 a\right) V_{2} .
$$

Thus in order to ensure that $\dot{V}_{2}$ is negative, $S$ has to satisfy:

$$
\begin{gathered}
\frac{K}{M} \Omega \underline{\gamma}\left(\frac{P_{1}}{L+y}+\frac{P_{2}}{L-y}\right)|S|^{\alpha} \geq \\
\left(\frac{\bar{\rho} \theta}{\rho a}\left[a^{2}+a b+b+\frac{k \bar{A}}{M}\left(\frac{P_{1}}{L+y}+\frac{P_{2}}{L-y}\right)\right]+a\right)|S|
\end{gathered}
$$

and consequently, $K|S|^{\alpha} \geq \Gamma|S|$ where $\Gamma$ is given by (8). By noticing that $|u|=K|S|^{\alpha} \leq U$ one has to choose $|S|<$ $\bar{S}$ where $\bar{S}=\frac{U}{\Gamma}$ which leads to the third inequality in (9). The satisfaction of (16) leads to $V_{1}<\left(\frac{\theta \bar{\rho} U}{\sqrt{\underline{\underline{\rho}}} a \Gamma}\right)^{2}$, the second inequality in (9). Finally, $K \bar{S}^{\alpha}=U$ and $\bar{S}=\frac{U}{\Gamma}$ leads to $K=$ $\Gamma^{\alpha} U^{1-\alpha}$.

Remark 4: In order to evaluate the validity of the attraction domain given by (9), set $a=10$ and $b=40$, which gives $\bar{\rho}=40$ and $\rho=1$. Taking $\gamma=0.2, \theta=1.1$ leads to $\Gamma \approx 9$. Then from the second equation of (9), one gets, $y_{\max }<0.1 \mathrm{~m}$ and $\dot{y}_{\max }<0.7 \mathrm{~m} / \mathrm{s}$ which corresponds, roughly, to the half of the maximal velocity authorized by the constructor $(1.5 \mathrm{~m} / \mathrm{s})$. In addition, from the third inequality of (9) one gets $\ddot{y}_{\max }<$ $\frac{U}{\Gamma}+a \dot{y}_{\max }+b y_{\max }$ leading to $\ddot{y}_{\max } \leq 11 \mathrm{~m} / \mathrm{s}^{2}$.

Note that remark 4 is of main importance since the calculated bounds will be validated experimentally in section VI.

\section{B. Time varying perturbation $(|\dot{\Delta}| \geq 0)$}

It is shown in the present section that under boundedness assumptions on $\Delta$ and $\dot{\Delta}$ ( $\dot{\Delta}$ can be discontinuous), the closed loop system converges inside a ball around the origin, the radius of the ball can be reduced by decreasing $\alpha$. In particular, with $\alpha=0$, the closed loop transforms into a variable structure system which is insensitive to the perturbation. This is presented in the following proposition:

Proposition 4.1: Let assumptions 1 and 2 be satisfied and assume, in addition, that $\Delta$ is a differentiable function, such that $|\sup \dot{\Delta}|=D, D<\frac{2 \Omega \gamma P_{a} U}{L}$. Consider the closed loop system (6), (10) initialized inside the domain delimited by:

$$
\left\{\begin{array}{ccc}
\left|y_{0}\right| & \leq c-l, \\
Y_{0}^{\prime} \mathcal{P} Y_{0} & < & {\left[\frac{\theta \bar{\rho}}{\sqrt{\underline{\rho}} a}\left(U-\frac{D L}{2 \Omega \underline{\gamma} P_{a}}\right) \frac{1}{\Gamma}\right]^{2}} \\
\left|\ddot{y}_{0}+a \dot{y}_{0}+b y_{0}\right| & < & \left(U-\frac{D L}{2 \Omega \underline{\gamma} P_{a}}\right) \frac{1}{\Gamma}
\end{array}\right.
$$

where $a, b, \underline{\rho}, \bar{\rho}, \theta$ and $\Gamma$ are taken according to theorem 4.1.

Then for any $\alpha$ such that: $0<\alpha<1-\frac{D L}{2 \Omega \gamma P_{a} U}$, there exist a ball $B_{\delta}$, centered at the origin (i.e $Y=0, \ddot{y}=0$ ), of radius $\delta$, such that any trajectory of (6), (10), initialized according to (18), converges inside $B_{\delta}$ where $\delta$ is bounded from above by $\delta \leq r=\left(\frac{D L}{2 \Omega \underline{\gamma} P_{a} K(1-\alpha)}\right)^{\frac{1}{\alpha}}$. The gain $K$ in (10) is given by $K=\Gamma^{\alpha} U\left(U-\frac{D L}{2 \Omega \underline{\underline{\gamma} P_{a}}}\right)^{-\alpha}$. In addition, if $\alpha=0$, the control

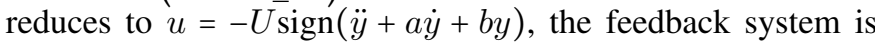
insensitive to the perturbation and converges asymptotically to zero.

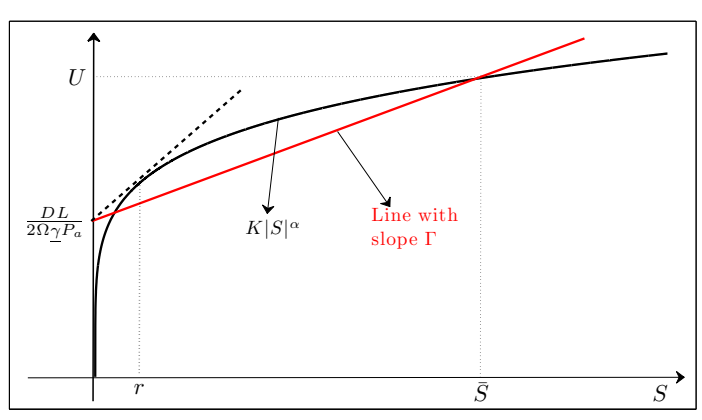

Fig. 3. Representation of $K|S|^{\alpha}$ and $\Gamma|S|+\frac{D L}{2 \Omega \underline{\gamma} P_{a}}$

Proof: Here again, the proof is carried out using (7). With a non constant perturbation, it can be verified that the inequalities (15) and (17) can be reduced respectively to:

$$
\begin{aligned}
\dot{V}_{2} \leq-\frac{K}{M} \Omega & \underline{\gamma}\left(\frac{P_{1}}{L+y}+\frac{P_{2}}{L-y}\right)|S|^{\alpha+1}+a b|y||S|+a|S|^{2}+\frac{D|S|}{M} \\
+ & {\left[a^{2}+b+\frac{k \bar{A}}{M}\left(\frac{P_{1}}{L+y}+\frac{P_{2}}{L-y}\right)\right]|\dot{y}||S| }
\end{aligned}
$$

and

$$
\begin{gathered}
\dot{V}_{2} \leq-2^{\frac{\alpha+1}{2}} \frac{K}{M} \Omega \underline{\gamma}\left(\frac{P_{1}}{L+y}+\frac{P_{2}}{L-y}\right) V_{2}^{\frac{\alpha+1}{2}}+\frac{D}{M} \sqrt{2 V_{2}}+ \\
\left(\frac{2 \bar{\rho} \theta}{\rho a}\left[a^{2}+a b+b+\frac{k \bar{A}}{M}\left(\frac{P_{1}}{L+y}+\frac{P_{2}}{L-y}\right)\right]+2 a\right) V_{2} .
\end{gathered}
$$

Consequently, in order to ensure that $\dot{V}_{2}$ is negative, the inequality (20) is reduced, with the aid of (14) to the following one:

$$
\begin{gathered}
\frac{K}{M} \Omega \underline{\gamma}\left(\frac{P_{1}}{L+y}+\frac{P_{2}}{L-y}\right)|S|^{\alpha} \geq \\
\left(\frac{\bar{\rho} \theta}{\rho a}\left[a^{2}+a b+b+\frac{k \bar{A}}{M}\left(\frac{P_{1}}{L+y}+\frac{P_{2}}{L-y}\right)\right]+a\right)|S|+\frac{D}{M} .
\end{gathered}
$$

Notice that (21) follows if the following inequality is verified:

$$
K|S|^{\alpha} \geq \Gamma|S|+\frac{D L}{2 \Omega \underline{\gamma} P_{a}},
$$

where $\Gamma$ is given by (8). The function $K|S|^{\alpha}$ and the straight line $\Gamma|S|+\frac{D L}{2 \Omega \gamma P_{a}}$ are depicted in figure 3 in order to simplify the proof. With the aid of figure 3 and by noticing that $K|S|^{\alpha} \leq U, D$ (and consequently $\dot{\Delta}$ ) should be sufficiently small in order to satisfy $\frac{D L}{2 \Omega \gamma P_{a}}<U$. Secondly, by using figure 3 , one can notice that the following inequality has to be satisfied $\Gamma|S|+\frac{D L}{2 \Omega \underline{\gamma} P_{a}}<U$ leading to the last inequality in (18) $|S|<\bar{S}=\left(U-\frac{D L}{2 \Omega \underline{\gamma} P_{a}}\right) \frac{1}{\Gamma}$. In addition, (13) leads to the second inequality in $(1 \overline{8})$.

Moreover, and by using figure 3 once again, one has to choose $\alpha$ such that the slope of $K|S|^{\alpha}$ at $S=\bar{S}$ is less than $\Gamma$. This leads to $\alpha K|\bar{S}|^{\alpha-1}<\Gamma$ and consequently to the condition on the choice of $\alpha$ :

$$
\frac{\alpha U}{\left(U-\frac{D L}{2 \Omega \underline{\gamma} P_{a}}\right) \frac{1}{\Gamma}}<\Gamma \Rightarrow \alpha<1-\frac{D L}{2 \Omega \underline{\gamma} P_{a} U} .
$$


Moreover, using that $K \bar{S}^{\alpha}=U$ leads to $K=$ $\Gamma^{\alpha}\left(U-\frac{D L}{2 \Omega \underline{\gamma} P_{a}}\right)^{-\alpha}$. Finally, it remains to calculate the radius of the ball of convergence, that is the intersection of $K|S|^{\alpha}$ and $\Gamma|S|+\frac{D L}{2 \Omega \gamma P_{a}}$ in figure 3. An upper estimate can be given by the tangent to $K|S|^{\alpha}$ issued from $\frac{D L}{2 \Omega \gamma P_{a}}$ which corresponds to the point $r$ in figure 3 . For this aim, reconsider (22) and notice that the following inequality is verified: $K r^{\alpha}=\frac{K \alpha}{r^{1-\alpha}} r+\frac{D L}{2 \Omega \gamma P_{a}}$. Solving for $r$ leads to $r=\left(\frac{D L}{2 \Omega \underline{\gamma} P_{a} K(1-\alpha)}\right)^{\frac{1}{\alpha}}$. The proof of the last assertion of proposition is straightforward. It is proven from (21) by replacing $K|S|^{\alpha}$ by $U$ in the left hand side of the inequality.

\section{Robustness against parameter variation and modeling er- rors}

Previous sections IV-A and IV-B discussed the influence of a constant additive perturbation $(\dot{\Delta}=0)$ and a time varying one.

Let us assume that the parameters $T, A_{1}, A_{2}$ etc. can vary due, for example, to a mis-modeling or aging. Two cases have to be discussed. If $\dot{\Delta}=0$ then, according to theorem 4.1 , a parameter change influences only the parameter $\Gamma$ in (9) and consequently the size of the domain of convergence however, the asymptotic stability is still guaranteed. On the other hand, if $\dot{\Delta}$ is time varying, a parameter change influences both $D$ and $\Gamma$ in (18) and consequently both the domain of convergence (18) as well as the size of $\mathcal{B}_{\delta}$ are modified.

\section{NUMERICAL ISSUES: DERIVATIVE ESTIMATION}

In order to implement the controller, first and second order derivatives has to be available. They can be estimated using an observer or a differentiator. The literature about derivative estimation of noisy signals is large, a well known technique is based on the theory of sliding modes [9]. The robustness to noises and the low computational cost of the numerical differentiators of [11] motivated their use in the present work. Briefly, the method considers a Taylor expansion as a local model of the noisy signal, convolves it with a properly chosen polynomial in order to obtain the desired derivative estimate as the output of the convolution (i.e. a filtering operation). Afterward, the convolution formula is discretized using a numerical integration scheme in order to obtain a finite impulse response filter. The method is summarized in the following lines for first and second order derivatives. Note that derivative estimation of any order has also been provided in [11].

\section{A. First order derivative}

Consider a first order Taylor expansion:

$$
y(t-\mathcal{T} s)=y(t)-\mathcal{T} \dot{y}(t) s
$$

where $s$ is an idle variable and $\mathcal{T}$ a design parameter that corresponds to the length of a sliding window used for the estimation of the derivative. Multiplying both sides of (23) by $\frac{d}{d s}(1-s) s=1-2 s$ and integrating over $[0,1]$ with respect to $s$, leads to:

$\int_{0}^{1}(1-2 s) y(t-\mathcal{T} s) d s=y(t) \int_{0}^{1}(1-2 s) d s-\mathcal{T} \dot{y}(t) \int_{0}^{1} s(1-2 s) d s$.
After straightforward simplifications, a causal, first order, derivative estimator is obtained:

$$
\dot{y}(t)=\frac{6}{\mathcal{T}} \int_{0}^{1}(1-2 s) y(t-\mathcal{T} s) d s .
$$

\section{B. Second order derivative}

Consider now a second order Taylor expansion:

$$
y(t-\mathcal{T} s)=y(t)-\mathcal{T} \dot{y}(t) s+\frac{\mathcal{T}^{2} s^{2}}{2} \ddot{y}(t) .
$$

Multiplying both sides of (25) with $\frac{d^{2}}{d s^{2}}(1-s)^{2} s^{2}=2-12 s+$ $12 s^{2}$ and integrating over $[0,1]$ leads, after simplifications, to the following, causal, second order, derivative estimator:

$$
\ddot{y}(t)=\frac{60}{\mathcal{T}^{2}} \int_{0}^{1}\left(1-6 s+6 s^{2}\right) y(t-\mathcal{T} s) d s .
$$

\section{Discretization}

For the first order derivative estimator, the integral is numerically approximated using the trapezoidal rule. While for the second order derivative, Simpson rule has been used since it involves a higher order polynomial. The first order derivative estimator has been discretized using 9 samples. It is given by: $h_{1}=[4.6875,7.0313,4.6875,2.3438,0,-2.3438$, $4.6875,-7.0313,4.6875]$. Therefore the parameter $\mathcal{T}$ in (24) is of 0.08 seconds since the sampling time is $d=0.01$ second. The second order derivative estimator has been discretized using 21 samples $^{4}$. The one used in the experimental tests is the following. $h_{2}=[25,71.5,23,23.5,2,-12.5,-$ $13,-36.5,-22,-48.5,-25,-48.5,-22,-36.5$, $13,-12.5,2,23.5,23,71.5,25]$. Therefore the parameter $\mathcal{T}$ in (26) is of 0.2 second since the sampling time is $d=0.01$ second.

\section{EXPERIMENTATION}

The experimental setup that served for the experiments is depicted in figure 4. It can be shown the servovalve (Festo, MPYE-5-1-1/8-LF-010-B), the cylinder (Festo, DNCI32-200-P-A-MU) with a payload of $5 \mathrm{Kg}$, the compressor (silent compressor, C.I.F CS-25), the dS1103 with interface panel and the monitoring via ControlDesk. The control has been implemented using Matlab-Simulink. The sampling time $d$ is equal to $d=0.01$ second. This sampling time allows the implementation of the controller on low cost real-time boards. The control law is given by: $u_{j}=-K^{\prime} K \operatorname{sign}\left(\tilde{\ddot{y}}_{j}+a \tilde{\dot{y}}_{j}+\right.$ $\left.b y_{j}\right) \times\left|\tilde{\ddot{y}}_{j}+a \tilde{\dot{y}}_{j}+b y_{j}\right|^{\alpha}$ where $j$ denotes the present, discretetime, instant. The position signal first and second derivatives $(\tilde{\dot{y}}$ and $\tilde{y})$ are evaluated using the filters, $h_{1}$ and $h_{2}$, calculated in section $\mathrm{V}$ with the same sampling time $d$ as follows: $\tilde{\dot{y}}_{j}=\sum_{i=1}^{9}\left(h_{1}(i) \times y_{j+1-i}\right)$ and $\tilde{\ddot{y}}_{j}=\sum_{i=1}^{21}\left(h_{2}(i) \times y_{j+1-i}\right)$. The bloc diagram of the control law is shown in figure 5 . Due to the asymmetry induced by the piston rod, as well as the vertically mounted cylinder, the control gain $K^{\prime}$ in the upward direction has been set to $120 \%$ of the gain in the downward

\footnotetext{
${ }^{4}$ As the second order derivative is more sensitive to noise than the first order derivative, more samples are used to filter out the noise.
} 


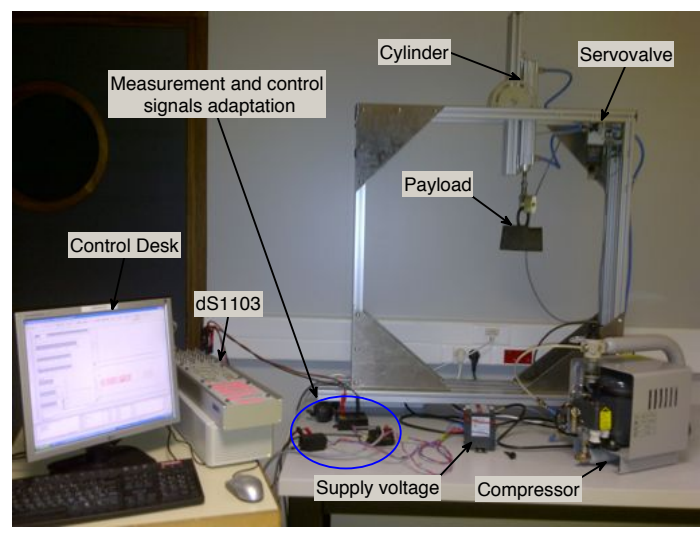

Fig. 4. Experimental setup

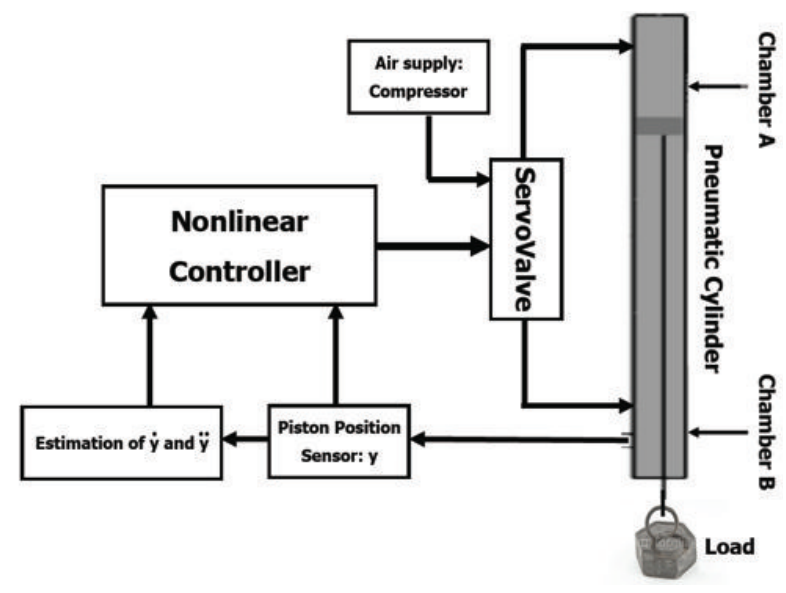

Fig. 5. Block diagram scheme of the proposed controller

direction. That is $K^{\prime}=1.2$ if $u_{k} \geq 0$ and $K^{\prime}=1$ if $u_{k}<0$. The parameters of the experimental setup are given in table I. A particularity of the benchmark is that the air compressor has a nominal power of $0.13 \mathrm{KW}$, a flow rate of $17 \mathrm{l} / \mathrm{min}$ at 0 bar and $12 l / \min$ at 8 bars. Roughly, the compressor can drive $13 \mathrm{Kg}$ at a velocity of $1 \mathrm{~m} / \mathrm{s}$. The servovalve nominal flow rate is $350 \mathrm{l} / \mathrm{min}$ and it is oversized compared to the compressor flow rate, as it will shown in the experimental results on the control magnitude. However, the compressor provide two advantages, firstly it is a silent one, secondly, it is easily transportable. The actuator accepts a maximal load of $15 \mathrm{Kg}$ when mounted in a vertical position. Therefore, in order not to overload neither the compressor nor the actuator, the tests has been conducted with a maximum payload of $11 \mathrm{Kg}$. Firstly, a stabilization experiment is presented in section VI-A in order to validate the domain of attraction computed in the theoretical part (see remark 4). Then the step response experiments of the cylinder are detailed in section VI-B where the value of $\alpha$ is taken in the set $\alpha \epsilon$ $\{0.1,0.5,0.8\}$ and the payload $\Delta$ is gradually increased as follows: $0,20,50,70,90,110$ Newtons. The load can be considered as slowly varying $(\dot{\Delta} \approx 0)$ due to the mechanical clearances between the payload and the piston rod eye (see figure 4). Except for $\alpha$, all the parameters $\left(a, b, h_{1}, h_{2}, K\right)$ are kept constant in the experimental tests.

In the coming experiments, one should notice that the transient responses are affected by both the load variation and the value of the parameter $\alpha$. Since the piston is mounted in a vertical position, increasing the load increases (resp. decreases) the time of an upward (resp. downward) displacement. In order to point out the influence of $\alpha$, let us take $0<\alpha_{1}<\alpha_{2}<1$ and notice that as $|S|<1$ then $|S|^{\alpha_{1}}>|S|^{\alpha_{2}}$, the control effort is bigger which offers a faster convergence. The opposite effect takes place if $|S|>1$.

\section{A. Bound validation}

The piston is charged with a mass of $2 K g$, as can be seen in figure 6 the initial conditions are $y_{0} \approx 0.09 \mathrm{~m}, \dot{y}_{0}=-0.53 \mathrm{~m} / \mathrm{s}$ and $\ddot{y}_{0}=0.5 \mathrm{~m} / \mathrm{s}^{2}$. They are initialized inside the physical domain given by remark 4 . Notice that during the transient all the variables remain inside the predefined bounds.

\section{B. Case 1: $\alpha=0.1$}

1) $\Delta=0$ Newton: In this experiment, the pneumatic cylinder is used without load. It can be noted that the piston position plotted in figure 7(a) follows the desired position with a static error close to zero in steady state condition (see zoom in figure 7(a)). The velocity and acceleration signals were not reported to due space limitation, however, they are estimated without noise and their maximal magnitudes are $1 \mathrm{~m} / \mathrm{s}$ and $6 \mathrm{~m} / \mathrm{s}^{2}$ respectively. The control input (figure 7(b)) exhibits chattering phenomena as can be obtained in case of conventional sliding mode. However, the chattering frequency is of $20 \mathrm{~Hz}$ approximately (see zoom in figure 7(b)), which is acceptable for the servovalve having a natural frequency of $100 \mathrm{~Hz}$.

2) $\Delta=50$ and $\Delta=70$ Newtons: Two experiments have been done with a payload of $5 K g$ and $7 \mathrm{Kg}$ respectively. Compared to the previous case VI-B1 (free load), the results were quite similar and consequently not reported herein.

3) $\Delta=90$ and $\Delta=110$ Newtons: In this experiment, the value of the load is again increased to reach $75 \%$ (11 $\mathrm{Kg}$ ) of the maximal load indicated by the constructor (see table I). By comparing to previous cases VI-B1 and VI-B2, a degradation of the performances can be noticed from figure 8(a) since an overshoot appeared when the piston is traveling downward. This results in an almost elimination of chattering as it can be seen in figure 8(b). Note that the results of the experiment with $9 \mathrm{Kg}$ weight are quite similar to the case $11 \mathrm{Kg}$, nevertheless, an overshoot with smaller amplitude is remarked. The stabilization time increases as the load $\Delta$ increases.

\section{Case 2: $\alpha=0.5$}

This experiment is conducted to study the influence of the parameter $\alpha$. Its value is increased to $\alpha=0.5$. The pneumatic cylinder is loaded with a $2 \mathrm{Kg}, 5 \mathrm{Kg}$ and $7 \mathrm{Kg}$ weights. Under $2 K g$ and $5 K g$, the controller performs well. However, as it can be remarked from figure 9 (a), under $7 \mathrm{Kg}$, the piston position follows its reference with a significant static 


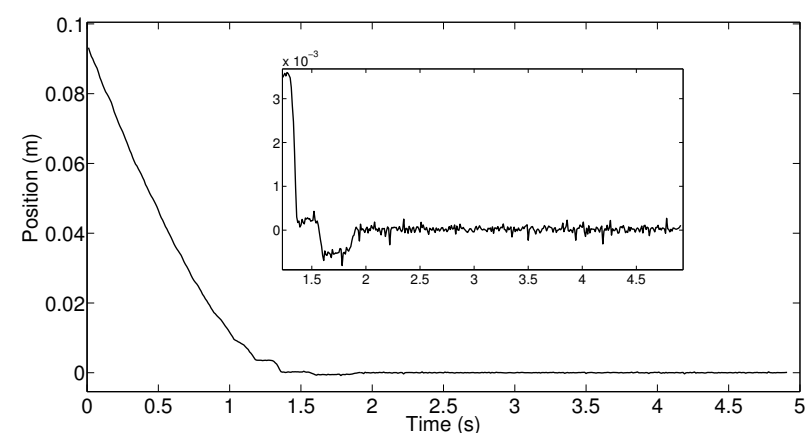

(a) Position

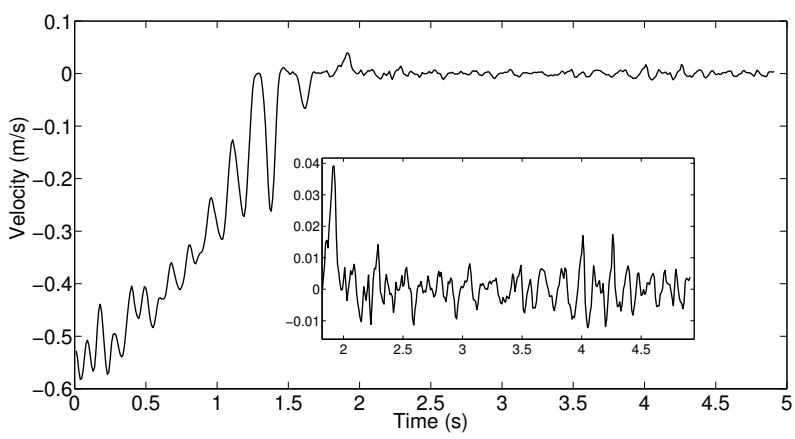

(b) Velocity estimate

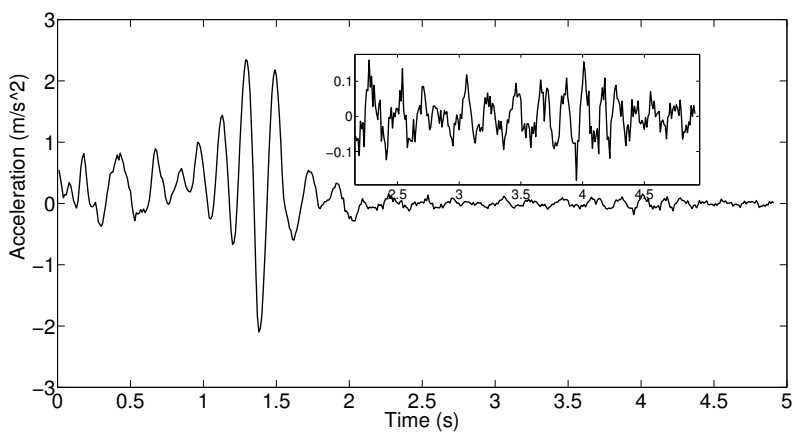

(c) Acceleration estimate

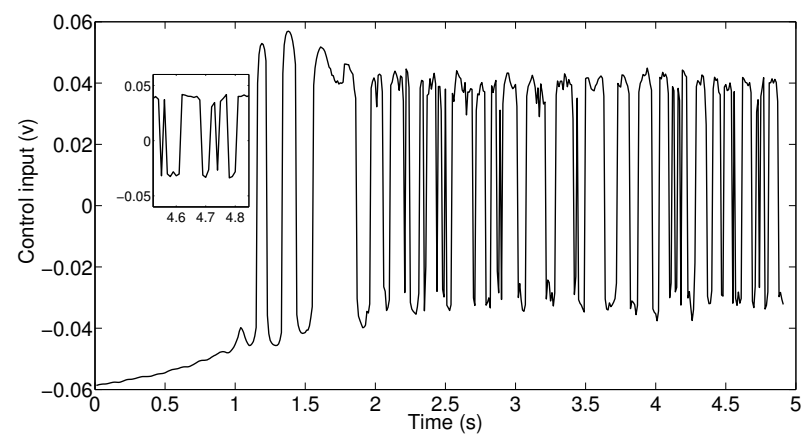

(d) Control input

Fig. 6. Validation of the domain of attraction of the controller

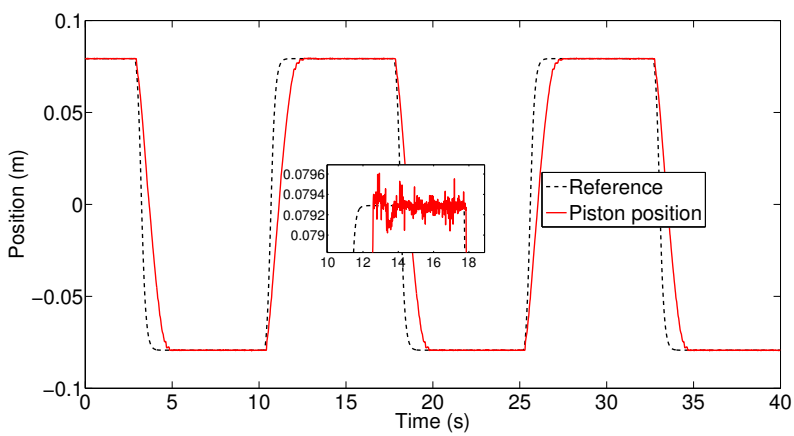

(a) Position

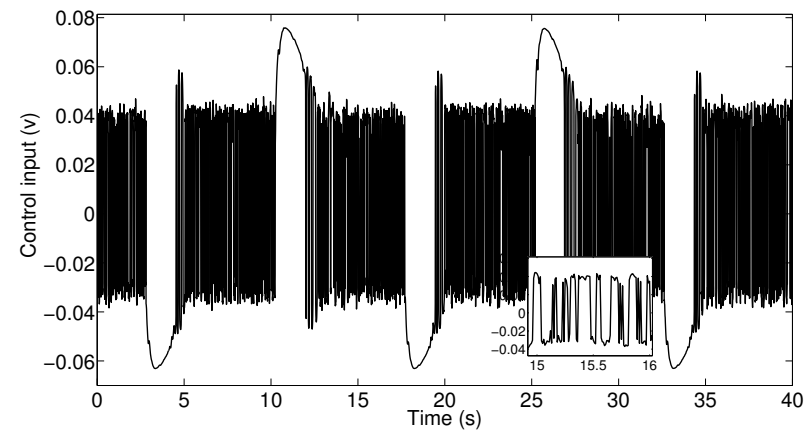

(b) Control input

Fig. 7. Free load experiment, $\alpha=0,1$.

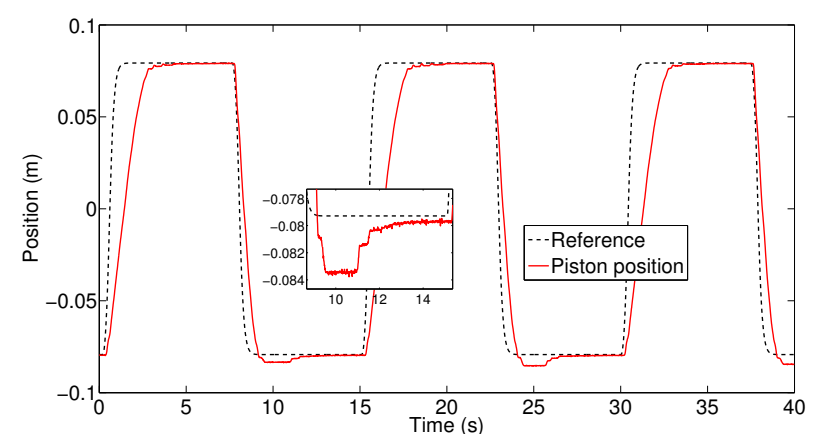

(a) Position

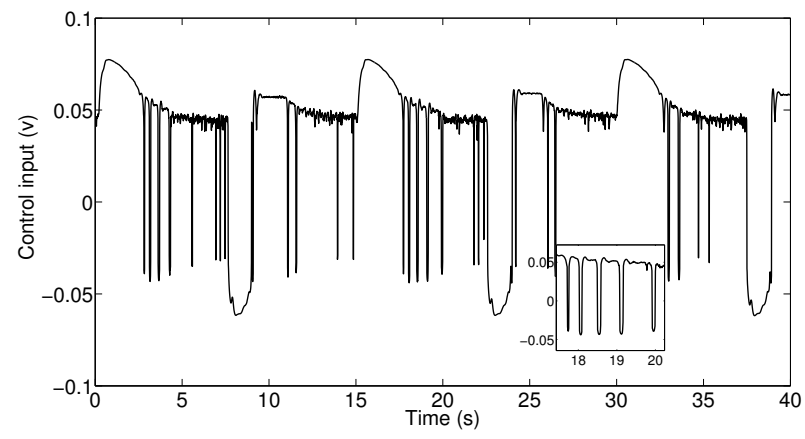

(b) Control input

Fig. 8. Experiment with $11 \mathrm{Kg}$ of load, $\alpha=0,1$. 


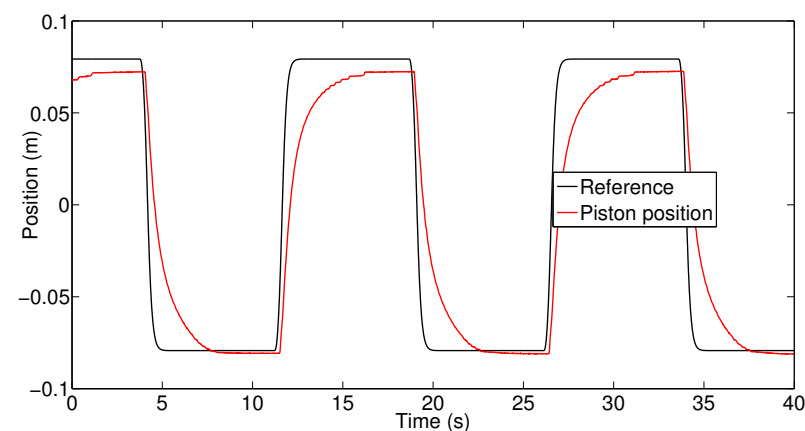

(a) Position

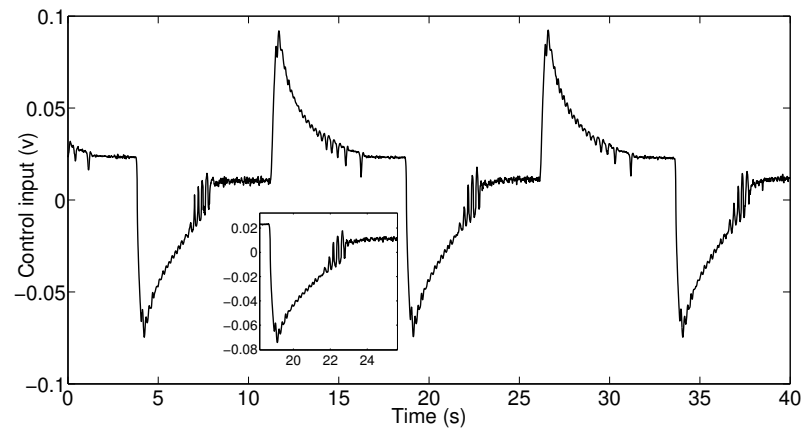

(b) Control input

Fig. 9. Experiment with $7 \mathrm{Kg}$ of load, $\alpha=0,5$.

error and a non symmetric behavior in step response. As a consequence, the chattering disappears to the detriment of a net loss in performances as it is obtained in the figure 9(b). The asymmetric static error can be caused by load and frictions acting in same direction in the upward displacement.

\section{Case 3: $\alpha=0.8$}

With $\alpha=0.8$, the controller provides a small static error for only the $2 \mathrm{Kg}$ load (see figure 10(a)). However, it induces an asymmetric step response in the upward and downward motions. When $\alpha$ is increased, it can be seen from figure 10 (b) that both the magnitude and the frequency $(\approx 8 \mathrm{~Hz})$ of the chattering phenomena are decreased. However, the performances are significantly altered by increasing the load.

\section{E. Comparison to previous works}

The previous works on pneumatic systems control recalled in section I-A can be classified in two categories, linear controllers [20] and nonlinear controllers [5], [12], [14], [18].

The control proposed in this work cannot be accurately compared to [5], [14], [18] since they all involve pressure sensors, nor to [12] since it uses a different technology of valves (solenoid valves instead of a servovalve).

The PID plus acceleration feedback $\left(\right.$ PIDD $\left.^{2}\right)$ controller proposed in [20] is implemented on our experimental setup in order to illustrate a structural defect of linear control in the presence of Coulomb frictions known as the "hunting effect" [7]. Since a tuning rule of the controller parameters $\left(K_{p}\right.$, $K_{i}, K_{d}$ and $K_{a}$ ) was not provided in [20], we proceeded as follows. Our first guess consisted of the parameters given by

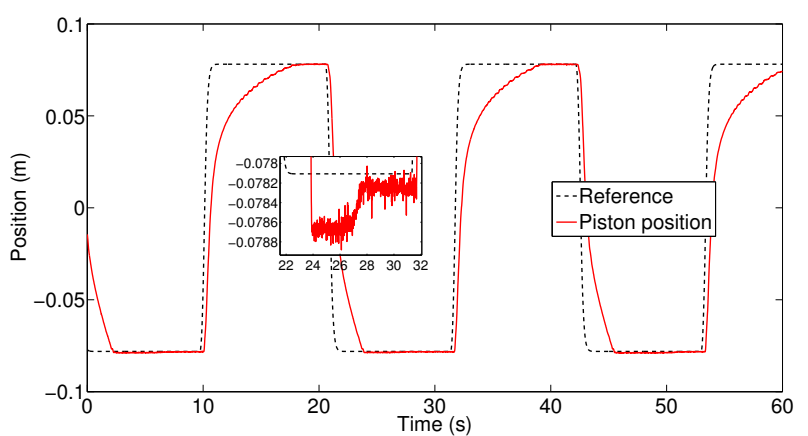

(a) Position

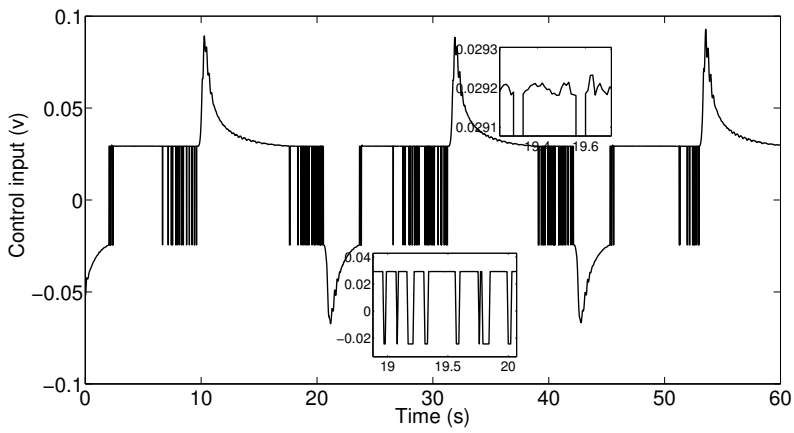

(b) Control input

Fig. 10. Experiment with a load of $2 K g, \alpha=0.8$.

[20] $K_{p}=0.03, K_{i}=0.01, K_{d}=0.3$ and $K_{a}=0.06$, then a trial and error procedure is employed in order to find an acceptable parameter set. The obtained values are $K_{p}=2.2$, $K_{i}=0.8, K_{d}=0.05$ and $K_{a}=0.001$. The velocity and acceleration are estimated using the numerical differentiators described in section $\mathrm{V}$. The controller is tuned under no load condition. Figures 11(a) and 11(b) show the results of an experiment under a $2 \mathrm{Kg}$ load clearly illustrating the hunting effect [7] caused by Coulomb frictions on the piston rod and the integral action of the controller. The overshoot which appears on the step response increased under the $2 \mathrm{Kg}$ load.

\section{CONCLUSION}

In this paper a state feedback nonlinear controller was proposed for a pneumatic cylinder by using the theory of homogeneous, finite time stable, ordinary differential equations. Based on Lyapunov theory, asymptotic stability of the closed loop system was proved and a domain of attraction of the controller was ensured. Theoretical analyses were confirmed through experimental tests using a pneumatic cylinder controlled by dSPACE dS1103 microcontroller. In fact, in order to increase the precision (decrease the static error), and obtain a symmetric behavior in the upward and downward step responses, the parameter $\alpha$ of the controller should be decreased. The main disadvantage is the chattering of the control signal. Theoretically, the control is a continuous function. Therefore, chattering should not appear. However, as $\alpha$ decreases the inevitable noise on the estimated velocity and acceleration is amplified resulting in a fluctuating control signal.

It is well known that the introduction of a delay in modeling 


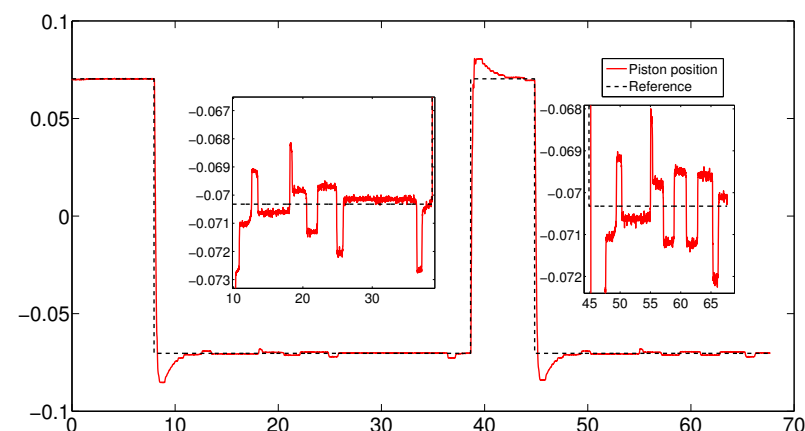

(a) Position

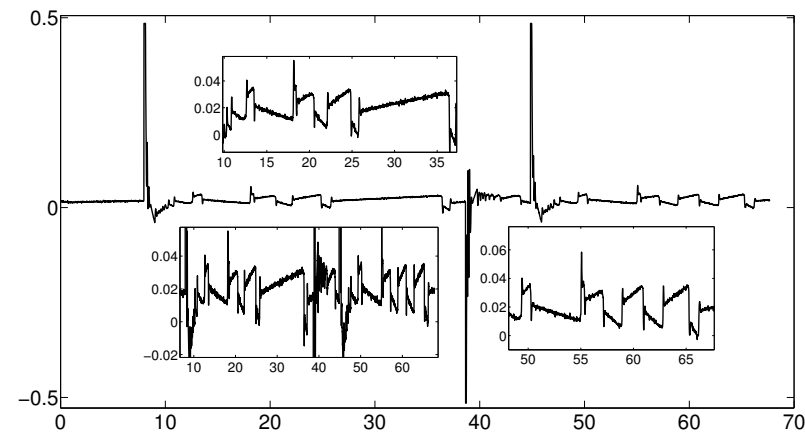

(b) Control input

Fig. 11. Experiment with a load of $2 \mathrm{Kg}$ for the controller of [20].

complex systems allows one to obtain a simple mathematical representation of the system [1]. Thus, it seems interesting to model the pressure dynamics through the introduction of a (variable) delay and design a controller based on a second order model having a delayed input. This possibility will be investigated in a future work and compared to the results obtained in the present one.

\section{Acknowledgment}

This work was supported by the Agregation Project under FUI Grant No. 10. We would like to thank anonymous reviewers for their useful comments, which have greatly improved the manuscript.

\section{REFERENCES}

[1] Astöm K. and Hägglund T., "PID controllers: Theory, Design and Tuning". ISA, $2^{\text {nd }}$ edition, 1995.

[2] Bhat P. and Bernstein D.S., "Finite time stability of homogeneous systems", Proceedings of the ACC, New Mexico, 1997.

[3] Bhat P. and Bernstein D.S., "Finite-Time Stability of Continuous Autonomous Systems". SIAM J. Control Optim. Vol. 38, No. 3, pp. 751-766, 2000.

[4] Bhat P. and Bernstein D.S., "Geometric homogeneity with applications to finite-time stability". Mathematics of Control, Signals and Systems Vol. 17, Issue 2, pp 101-127, 2005.

[5] Bone G.M. and Ning S., "Experimental Comparison of Position Tracking Control Algorithms for Pneumatic Cylinder Actuators". IEEE/ASME Transactions on mechatronics, Vol. 12, No. 5, pp. 557-561, 2007.

[6] Canudas de Wit C., Olsson H., Astrom K.J. and Lischinsky P., "A new model for control of systems with friction". Trans. on Automatic control Vol. 40, No. 3 pp 419-425, 1995.

[7] Hensen R.H.A., Van de Molengraft M.J.G. and Steinbuch M., "Friction induced hunting limit cycles: A comparison between theLuGreandswitchfrictionmodel". Automatica, Vol. 39, pp. 2131-2137, 2003.

[8] Khalil H.K., "Nonlinear systems". Prentice Hall, 2002.
[9] Levant A., "Higher order sliding modes, differentiation and output feedback control". Int. J. Control Vol. 76, No. 9/10 pp. 924-941, 2003.

[10] Messina A., Giannoccaro N.I. and Angelo Gentile A., "Experimenting and modelling the dynamics of pneumatic actuators controlled by the pulse width modulation (PWM) technique”. Mechatronics Vol. 15, pp. 859-881, 2005.

[11] Mboup M., Join C. and Fliess M., "Numerical differentiation with annihilators in noisy environment". Numerical Algorithms Vol. 50, No. 4 pp. 439-467, 2009.

[12] Nguyen T., Leavitt J., Jabbari F. and Borbow J.E., "Accurate slidingmode control of pneumatic systems using low cost solenoid valves". Transactions on mechatronics Vol. 12, No. 2 pp 216-219, 2007.

[13] Pandian S.R.,Takemura F., Hayakawa Y. and Kawamura S., "Pressure Observer-Controller Design for Pneumatic Cylinder Actuators". IEEE/ASME Transactions on mechatronics, Vol. 7, No. 4, pp. 490-499, 2002.

[14] Rao Z. and Bone G.M., "Nonlinear Modeling and Control of Servo Pneumatic Actuators". Trans. on control systems technology Vol. 16, No. 3, pp. 562-569, 2008.

[15] Richardson R., Plummer A.R., and Brown M.D., "Self-Tuning Control of a Low-Friction Pneumatic Actuator Under the Influence of Gravity". Trans. on control systems technology Vol. 9, No. 2, pp. 330-334, 2001.

[16] Schulte H. and Hahn H., "Fuzzy state feedback gain scheduling control of servo-pneumatic actuators". Control Eng. Pract. vol. 12, pp. 639-650, 2004.

[17] Tressler J.-M., Clement T., Kazerooni H. and Lim M. "Dynamic behavior of pneumatic systems for lower extremity extenders.". Proceedings of IEEE international conference on robotics and automation, pp. 32483253, 2002.

[18] Tsai Y.-C. and Huang A.-C., "Multiple-surface sliding controller design for pneumatic servo systems". Mechatronics No. 18 pp. 506-512, 2008.

[19] Utkin V.I., Guldner J. and Shi J., "Sliding mode control in electromechanical systems". Taylor\& Francis London 1999.

[20] Wang J., Pu J. and Moore P., "A practical control strategy for servopneumatic actuator systems". Control Engineering Practice No.7 pp. $1483-14881999$. 